\title{
Astronomy Education in Universities of China
}

\author{
Cheng Fang and Yuhua Tang \\ Department of Astronomy, Nanjing University, Nanjing, China. e-mail: \\ fangc@nju.edu.cn
}

\begin{abstract}
In China, more than twenty universities have astronomy education and research work. In four key universities, a complete series of educational programs of undergraduate, master's, doctoral and postdoctoral levels has been formed. After four-year study at the undergraduate stage, students can be enrolled in master degree specialties. Three years later, some of them begin their three-year Ph.D. education. Only a few students enter into post-doctoral programs. Master's and doctoral education systems are also established in the Chinese Academy of Sciences. In this paper, we give a description of the astronomy education of universities in China. After introducing the overall situation, we describe teaching materials, graduate-degree courses and facilities. We also discuss some problems and prospects for the new century.
\end{abstract}

\section{Introduction}

After a rapid development in the 1990s, more than twenty universities in China have established astronomy education and research work. In these universities, there are courses of astronomy for the students in astronomy departments and/or over the whole university. Some groups of student amateur astronomers have been organized. Besides, since the end of 1970 s, astronomy education at M.S. and Ph.D levels has also been developed in the Chinese Academy of Sciences (CAS). Unlike the situation in some other countries, students can get their M.S. and Ph.D degrees in CAS. Nowadays, more than one third of M.S. and Ph.D students come from CAS. However, the education system is more or less similar to that in universities. Thus, here we will concentrate on astronomy education in universities in mainland China.

Among the twenty universities, there are four universities that are key ones and have relatively long histories. After a description of the astronomy departments in these four key universities in Section 2, the astronomical education system is described in detail in Section 3. The astronomical activities of students are illustrated in Section 4, followed by a brief discussion of the problems and prospects of the education system in Section 5.

\section{Astronomy Departments in Four Key Universities}

There are four key universities, namely, Nanjing University (NU), Beijing Normal University (BNU), Beijing University (BU) and University of Science and 
Technology of China (USTC), in which astronomy departments (AD) or an astronomy specialty (AS) or Center for Astrophysics (CFA) have been established for a shorter or longer time. Table 1 gives some information about them.

Table 1. Astronomy Departments in Four Key Universities

\begin{tabular}{lccccc}
\hline University & $\begin{array}{c}\text { Date of } \\
\text { Set Up }\end{array}$ & $\begin{array}{c}\text { No. of } \\
\text { Prof. }\end{array}$ & $\begin{array}{c}\text { No. of } \\
\text { Underg. }\end{array}$ & $\begin{array}{c}\text { No. of } \\
\text { M.S. }\end{array}$ & $\begin{array}{c}\text { No. of } \\
\text { Ph.D. }\end{array}$ \\
\hline AD of NU & 1952 & 18 & 80 & 20 & 10 \\
AD of BNU & 1960 & 5 & 70 & 15 & 10 \\
CFA of USTC* & 1978 & 7 & 20 & 10 & 9 \\
AS of BU** & 1960 & 3 & 30 & 14 & 4 \\
\hline Total & & $\mathbf{3 3}$ & $\mathbf{2 0 0}$ & $\mathbf{5 9}$ & $\mathbf{3 3}$ \\
\hline
\end{tabular}

* Since 1999, an astronomy department has been established.

** In 2000, an astronomy department has been established.

The main facilities for student training in these universities are as follows:

1. $60-\mathrm{cm}$ solar tower telescope (NU);

2. $65-\mathrm{cm}$ reflector $(\mathrm{NU})$;

3. 40-cm Schmidt-Cassegrain telescopes (NU and BNU);

4. 40-cm reflector (BNU);

5. 28-cm refractor (NU);

6. Radio telescopes ( $3 \mathrm{~cm}$ in NU, $2 \mathrm{~cm}$ in $\mathrm{BNU})$;

7. $15-\mathrm{cm}$ refractors ( $\mathrm{NU}$ and $\mathrm{BNU}$ );

8. PC and Sun workstations (NU, BNU, USTC and BU).

As an example, Figure 1 shows the picture of the solar-tower telescope in Nanjing University. It is the only one of its kind in China. Figure 2 shows the picture of the $40 \mathrm{~cm}$ reflector in BNU. Generally, students use these facilities to do some practical training. However, once time in the four-year study, students also go to astronomical observatories and spend about 2-3 weeks workimg under the direction of researchers. This is very useful for the students to know the recent research in progress.

A complete series of educational programs of undergraduate, master's, doctoral and post-doctoral levels has been formed since the 1950s, and highly improved in recent years.

\subsection{Undergraduate education}

Based on a national examination, students will be enrolled in universities according to their scores. There is a strong competition for enrollment in the universities. Each year, about 50 students are admitted to astronomy departments. After entry to university, four-year undergraduate education begins. 

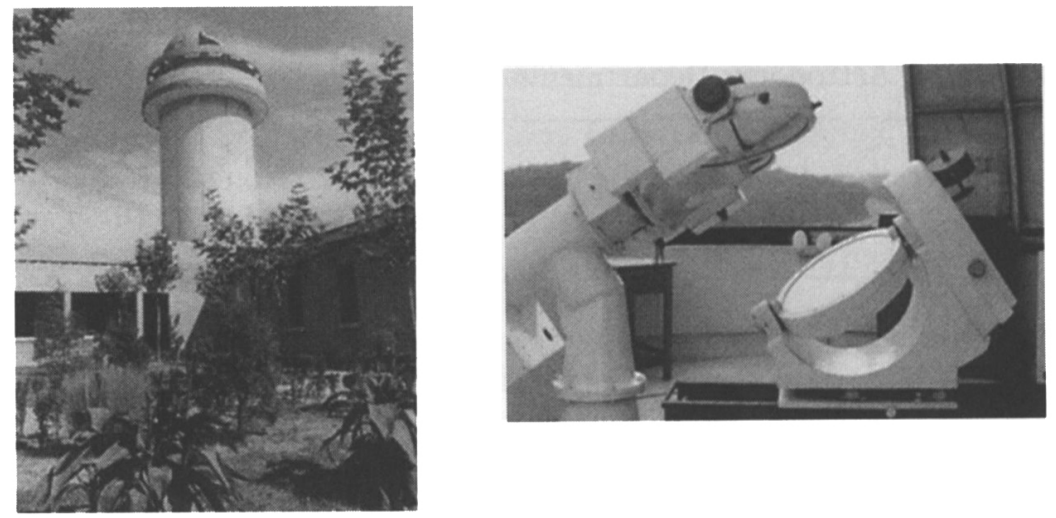

Figure 1. The 60-cm solar-tower telescope of Nanjing University (left) and its coelostats (right). This is the only instrument of its kind in China

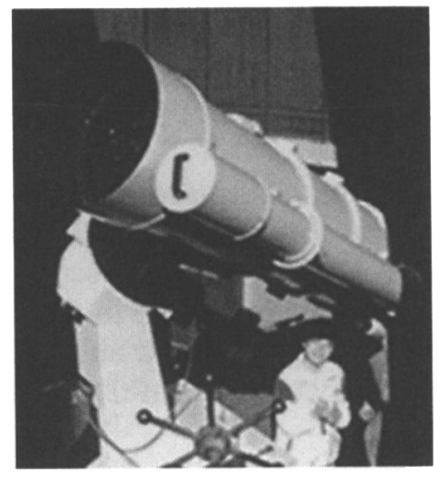

Figure 2. The 40-cm reflector of Beijing Normal University 
The universities have been perfecting the credit system, and have introduced the major-auxiliary course program and the dual bachelor's degree system.

During four years, students have to obtain 150 credits, among them $60 \%$ should be for the important necessary courses. In astronomy departments, all students should mainly study mathematics and physics during the first two years. The main astronomy courses during the next two years are as follows:

1. General astronomy;

2. Theoretical astrophysics;

3. Practical astrophysics;

4. Celestial mechanics;

5. Astrometry.

\subsection{Master's-degree specialties}

Students can be enrolled in the master's-degree specialty through recommendation, which is only for few excellent students, or by passing successfully the university examination. In recent years, there are about 30-35 students over the whole of China being admitted to astronomy master's-degree specialties of the universities. They have to spend 2.5-3 years to study courses of the master'sdegree program. The main courses are as follows:

1. Radiation mechanisms*;

2. Stellar interier structure and evolution*;

3. High energy astrophysics;

4. Plasma dynamics;

5. Radio astrophysics;

6. Galactic structure and evolution;

7. Physics of cosmology;

8. Physics of solar active regions;

9. Numerical simulation in astrophysics;

10. Molecular astrophysics.

The courses with * can be chosen by students.

The students should obtain certain credits (30-35) and publish at least one scientific paper during the three-year period. Having passed successfully the defense of their theses, students can get their M.S. degrees.

\subsection{Ph.D program}

Based on university examination and oral test, M.S. degree students can be enrolled in Ph.D programs. In recent years, there are about 15-20 students being admitted each year in the astronomy Ph.D programs of the universities. They have to spend 2.5-3 years studying a few special courses, such as:

Physics of $\gamma$-ray bursts;

Pulsar physics;

Supernovae and supernovae remnants;

Physics of compact stars;

Observational cosmology; 


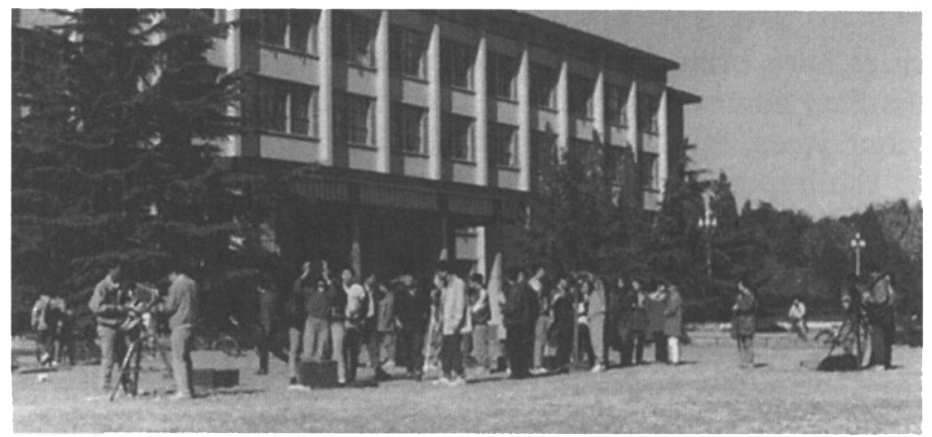

Figure 3. Observation of a partial solar eclipse organized by the student astronomical society of Beijing University

Physics of active galactic nuclei etc.

However, the main requirement in the Ph.D program is to do research work. Students have to publish between one and three scientific papers in the main astronomical journals. By the end of study, a student must write a comprehensive thesis, which has to be evaluated by about ten scientists, and well pass the defense of the thesis, in order to obtain the Ph.D degree.

\subsection{Post-doctoral program}

Based on application and competition, only a few $\mathrm{Ph}$.D students can be accepted in the post-doctoral program. Generally, it takes $1.5-2$ years and universities provide special funds and accommodations for them. The people in the postdoctoral program can do any research work they like, but they must provide a report by the end of the period of study.

\section{Astronomical Activities}

Some astronomical societies for young students have been organized in universities. They attract not only students in the astronomy departments, but also many amateur-astronomer students. A variety of astronomical activities, such as lectures, observations of celestial phenomena and popularization of astronomy etc., have been launched. As an example, Figure 3 shows the observation of a partial solar eclipse organized by the young student astronomical society of Beijing University.

Students in the astronomy departments participate frequently in society activities. They contribute to the popularization of astronomy, including organization of astronomical lectures, responses to inquiries about problems in astronomy raised by people etc. Figure 4 shows one such society activity.

\section{Problems and Prospects}

In recent years astronomy education in China has been greatly improved. It should be mentioned that, besides the universities, there is also an education 


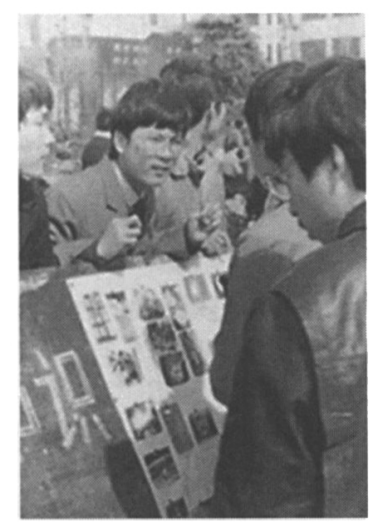

Figure 4. Responding to inquiries of people in a square in Nanjing

system of graduate students in the Chinese Academy of Sciences (CAS). Every year there are about 20-30 graduate students obtaining astronomical M.S. degrees in $\mathrm{CAS}$, while about 10 students obtain astronomical $\mathrm{Ph} . \mathrm{D}$ degrees in CAS. Thus, we have sources of well-educated students for astronomy. However, one problem is the loss of quality young people. After getting M.S. degrees, some excellent young students go abroad, especially to the United States, to enrol in Ph.D programs. Unfortunately, After obtaining their Ph.D degrees, many of them have no chance to get astronomical jobs. This is probably a common problem in developing countries. It is hoped that this situation will be improved in future.

Now we place great emphasis on basic scientific research and teaching and consolidate the student's theoretical foundation and astronomical surveying skills. Special training is offered to those students of greater creativity in an attempt to turn them into quality personnel. While continually reforming the graduate-degree courses, we have adopted the modes of running consecutive undergraduate and master's, master's and doctoral, or undergraduate, master's and doctoral programs. Interdepartmental, inter-institutional, and international joint training programs have been offered to students. Especially joint programs between universities and the CAS have been recently strengthened. Two astrophysical centers have been established: one is in Beijing University as a joint program between BU and the CAS and the other is in Nanjing as a joint organization between NU, USTC, the Purple Mountain Observatory and the Shanghai Observatory.

In summary, a complete series of educational programs of undergraduate, master's, doctoral and post-doctoral levels has been much improved in China. It provides a continuous good source for Chinese astronomers engaging in teaching, research and popularization of astronomy. We would like very much to learn the experience of foreign countries, especially of developing countries and to develop further the education system in China. 
Acknowledgments. We would like much to thank Professors Qiao Guojun, Ma Wen-zhang and Zhang Yang for their help in providing good data. This work was supported by a fund from the Doctoral Program of the Ministry of Education of China.

\section{Discussion}

Orchiston and Raharto asked if Chinese graduates in astronomy could find jobs in astronomy. Fang replied that there is no difficulty for those with doctorates. For those with only master's degrees, employment depended on their own choice and ability. Kochhar asked if Chinese students returning from the West experienced any difficulties in re-adjusting to their home culture. Fang thought not, except for some who stayed abroad for a long time. He believed that most young Chinerse scientists valued their home culture and could make any necessary adjustments themselves. Martinez observed that the fact that some excellent scientists migrated abroad was not entirely bad. Some of them will eventually return, bringing with them experience of areas of astronomy not represented in the home country. They will also bring their own network of contacts, thus expanding the influence of the home country's astronomy. Leeuw suggested that countries like China, India and South Africa (naming only those who had reported here) could do more to employ their young citizens with astronomy degrees from abroad, who could prove to be valuable new blood. Fang agreed with both the foregoing comments, stating that the Chinese suthorities are not opposed to students going abroad. They did hope some would return and measures are now being taken to encourage them to do so, including creating opportunities for temporary returns (upto some months). 